\section{Was the exacerbation of PTSD symptoms after high doses of venlafaxine due to the noradrenergic mechanism? A case report}

\author{
A piora dos sintomas de PTSD com o uso de \\ doses altas de venlafaxina deveu-se aos \\ mecanismos noradrenérgicos? Informe de \\ um caso
}

\section{Dear Editor,}

Post-traumatic stress disorder (PTSD) is an anxiety disorder that can arise after exposure to a terrifying event/ordeal in which severe physical harm occurred or was about to happen. PTSD symptoms include persistent re-experience of the trauma, avoidance of trauma-associated stimuli, and hyperarousal. ${ }^{1}$ In the present report, we describe a woman with PTSD whose symptoms were exacerbated by the use of high doses of venlafaxine.

\section{Case report}

Ms. LCD, a 45-year-old white woman with chronic severe PTSD since she was assaulted and battered three years ago. Fluoxetine (40 mg/day) was successfully prescribed to control symptoms such as hypervigilance, constant anxiety, fearfulness, feelings of detachment, sporadic flashbacks of the assault, and social isolation. However, following her son's death she developed a comorbid DSM-IV diagnosis of major depression, which symptoms included amotivation, anhedonia, anergy and suicidal ideation. Imipramine, initially, and paroxetine plus psychotherapy were substituted for fluoxetine, with minimal improvement. Venlafaxine was then introduced and, at the beginning, it was slightly helpful. However, after twelve days, while receiving a daily dose of $225 \mathrm{mg}$, her symptoms worsened, and there was severe exacerbation of PTSD symptoms, including irritability, affective lability, nightmares, frequent flashbacks and intrusive thoughts regarding the assault as well as extreme hypervigilance even though she was at home. Her depressive symptoms remained unchanged. Thereafter, the daily dose of venlafaxine was gradually reduced to $75 \mathrm{mg} /$ day over three days, and we observed a significant reduction of her PTSD symptoms.

\section{Discussion}

Hyperfunction of the sympathoadrenal axis associated with a reduced activity of the hypothalamic-pituitary-adrenal axis in patients with PTSD has been previously demonstrated. ${ }^{2-3}$ Support for noradrenergic dysregulation in patients with PTSD has included high 24-hour urinary epinephrine and norepinephrine excretion, high 24-hour plasma norepinephrine, a lower than normal number of platelet $\alpha_{2}$ adrenergic receptors, exaggerated cardiovascular and 3 methoxy-4-hydroxyphenylglycol (a norepinephrine metabolite) responses to intravenous yohimbine and high levels of norepinephrine in the cerebrospinal fluid. In addition, adrenergic agents such as the $\alpha_{2}$-adrenoreceptor agonists clonidine and guanfacine, and the $B$-adrenoreceptor agonist propranolol, all reduce sympathetic arousal and are effective in treating the hyperarousal, reexperiencing, and impulsivity seen in PTSD. ${ }^{3-4}$
Venlafaxine, a bicyclic antidepressant, is usually categorized as a serotonin-norepinephrine reuptake inhibitor, but it has been referred to as a serotonin-norepinephrine-dopamine reuptake inhibitor. At low and medium dosages, venlafaxine diminishes serotonin reuptake alone, similarly to a selective serotonin reuptake inhibitor. At higher dosages (from about $225 \mathrm{mg} /$ day), venlafaxine inhibits the reuptake of norepinephrine as well as that of serotonin. Furthermore, at even high dosages (starting around $300 \mathrm{mg} /$ day), it inhibits dopamine reuptake in addition to serotonin and norepinephrine.

Despite the evident limitations of our study (case report), the reported data presented here suggests that the noradrenergic effects of higher doses of venlafaxine were responsible for the PTSD symptom recrudescence, supporting the evidence of an overly sensitive noradrenergic system underlying some PTSD symptoms. ${ }^{4}$ More studies are necessary to establish and confirm this hyphotesis.

Felipe Filardi da Rocha, Humberto Correa Department of Psychiatry and Department of Pharmacology, Universidade Federal de Minas Gerais (UFMG), Belo Horizonte (MG), Brazil

Financing: None

Conflict of interests: None

\section{References}

1. Davidson JR. Pharmacologic treatment of acute and chronic stress following trauma. J Clin Psychiatry. 2006;67(Suppl 2):34-9.

2. Liberzon I, Abelson JL, Flagel SB, Raz J, Young EA. Neuroendocrine and psychophysiologic responses in PTSD: a symptom provocation study. Neuropsychopharmacology. 1999;21(1):40-50.

3. Southwick SM, Bremner JD, Rasmusson A, Morgan CA, Arnsten A, Charney DS. Role of norepinephrine in the pathophysiology and treatment of posttraumatic stress disorder. Biol Psychiatry. 1999;46(9): 1192-204.

4. Deneys ML, Ahearn EP. Exacerbation of PTSD symptoms with use of duloxetine. J Clin Psychiatry. 2006;63(3):496-7. 